\title{
Option Pricing in a Fractional Brownian Motion Environment
}

\author{
Ciprian Necula \\ Academy of Economic Studies \\ Bucharest, Romania
}

Email: cipnec@yahoo.com

This draft: February 12, 2002

\begin{abstract}
$\underline{\text { Abstract }}$
The purpose of this paper is to obtain a fractional Black-Scholes formula for the price of an option for every $t \in[0, T]$, a fractional Black-Scholes equation and a risk-neutral valuation theorem if the underlying is driven by a fractional Brownian motion $B_{H}(t), 1 / 2<H<1$. For this purpose we will first prove some results regarding the quasi-conditional expectation, especially the behavior to a Girsanov transform. We will also compare our results with the classical results based on the standard Brownian motion and we conclude that in the case of the fractional Brownian motion the price of the option no longer depends only on $T-t$.
\end{abstract}

\section{Introduction}

If $0<H<1$ the fractional Brownian motion ( $\mathrm{fBm}$ ) with Hurst parameter $H$ is the continuous Gaussian process $\left\{B_{H}(t), t \in \mathbf{R}\right\}, B_{H}(t)=0$ with mean $E\left[B_{H}(t)\right]=0$ and whose covariance is given by:

$$
C_{H}(t, s)=E\left[B_{H}(t) B_{H}(t)\right]=\frac{1}{2}\left\{|t|^{2 H}+|s|^{2 H}-|t-s|^{2 H}\right\}
$$

If $H=\frac{1}{2}$ then $B_{H}(t)$ coincides with the standard Brownian motion $B(t)$.

The fractional Brownian motion is a self-similar process meaning that for any $\alpha>0 \quad B_{H}(\alpha t)$ has the same law as $\alpha^{H} B_{H}(t)$. 
The constant $\mathrm{H}$ determines the sign of the covariance of the future and past increments. This covariance is positive when $H>\frac{1}{2}$, zero when $H=\frac{1}{2}$ and negative when $H<\frac{1}{2}$.

Another property of the fractional Brownian motion is that for $H>\frac{1}{2}$ it has long range dependence in the sense that if we put

$$
r(n)=\operatorname{Cov}\left(B_{H}(1), B_{H}(n+1)-B_{H}(n)\right)
$$

then

$$
\sum_{n=1}^{\infty} r(n)=\infty
$$

The self-similarity and long-range dependence properties make the fractional Brownian motion a suitable tool in different applications like mathematical finance.

Since for $H \neq \frac{1}{2}$ the fractional Brownian motion is neither a Markov process, nor a semimartingale, we can not use the usual stochastic calculus to analyze it. Worse still after a pathwise integration theory for fractional Brownian motion was developed (Lin (1995), Decreusefond and Ustunel (1999)) it was proven that the market mathematical models driven by $B_{H}(t)$ could have arbitrage (Rogers (1997)). The fractional Brownian motion was no longer considered fit for mathematical modeling in finance. However after the development of a new kind of integral based on the Wick product ( Duncan, Hu and Pasik-Duncan (2000), Hu and Oksendal (2000) ) called fractional Ito integral, it was proved (Hu and Oksendal (2000)) that the corresponding Ito type fractional Black-Schools market has no arbitrage. In the same paper (Hu and Oksendal (2000)) a formula for the price of a european option at $t=0$ is derived.

The purpose of this article is to extend the formula for every $t \in[0, T]$. We obtain risk-neutral valuation formula and a fractional Black-Scholes equation. We will also analyze the sensitivity indicators.

This paper is organized as follows : in Section 2, we remind some results on fractional Ito integral, in Section 3 we prove some results regarding the quasiconditional expectation, especially the behavior to a Girsanov transform, in section 4 we apply these results in the study of the european options.

\section{Background}

In this section we will present some result we will need for the rest of the paper. For more aspects on these maters you may consult the fundamental papers concerning fractional Ito integral (Duncan, Hu and Pasik-Duncan (2000), Hu and Oksendal (2000) ).

For a fix $H, 1 / 2<H<1$ define:

$$
\phi_{H}(t, s)=H(2 H-1)|t-s|^{2 H-2}
$$

Let $f: \mathbf{R} \rightarrow \mathbf{R}$ measurable. Then $f \in L_{\phi}^{2}(\mathbf{R})$ if

$$
|f|_{\phi}^{2}:=\int_{R} \int_{R} f(s) f(t) \phi_{H}(t, s) d s d t<\infty
$$


Defining the inner product

$$
\langle f, g\rangle_{\phi}:=\iint_{R} f(s) g(t) \phi_{H}(t, s) d s d t
$$

we have that $\left(L_{\phi}^{2}(\mathbf{R}),\langle\cdot,\rangle_{\phi}\right)$ is a Hilbert space.

If $f \in L_{\phi}^{2}(\mathbf{R})$ define $\int_{R} f(t) d B_{H}(t):=\lim _{n \rightarrow \infty} \int_{R} f_{n}(t) d B_{H}(t)$ where

$$
f_{n}(t)=\sum_{i} a_{i}^{n} \chi_{\left[t_{i}, t_{i}+1\right)}(t) \rightarrow f(t)
$$

and

$$
\int_{R} f_{n}(t) d B_{H}(t):=\sum_{i} a_{i}^{n}\left(B_{H}\left(t_{i+1}\right)-B_{H}\left(t_{i}\right)\right)
$$

Lemma 1.1 (Ito isometry) If $f \in L_{\phi}^{2}(\mathbf{R})$ then $E\left(\int_{R} f(t) d B_{H}(t)\right)^{2}=|f|_{\phi}^{2}$ If $f \in L_{\phi}^{2}(\mathbf{R})$ define $\varepsilon(f):=\exp \left(\int_{R} f d B_{H}-\frac{1}{2}|f|_{\phi}^{2}\right)$

Lemma 1.2 The linear span of $\left\{\varepsilon(f), f \in L_{\phi}^{2}(\mathbf{R})\right\}$ is dense in $L^{2}(\mu)$ where $\mu$ is the probability law of $B_{H}$.

Let

$$
h_{n}(x)=(-1)^{n} e^{x^{2} / 2} \frac{d^{n}}{d x^{n}}\left(e^{-x^{2} / 2}\right)
$$

be the Hermite polynomials.

If $f \in L_{\phi}^{2}(\mathbf{R})$ we put that $\langle\omega, f\rangle=\int_{R} f(t) d B_{H}(t, \omega)$.

If $\alpha=\left(\alpha_{1}, \ldots, \alpha_{1}\right) \in I$, the set of all finite multi-indices of nonnegative integers, and $\left(e_{n}\right)_{n}$ an orthogonal basis in $L^{2}(\mu)$ (see Lemma 3.1 in Hu and Oksendal (2000) ) we denote:

$$
\mathrm{H}_{\alpha}(\omega):=h_{\alpha_{1}}\left(\left\langle\omega, e_{1}\right\rangle\right) h_{\alpha_{21}}\left(\left\langle\omega, e_{n}\right\rangle\right) \ldots h_{\alpha_{n}}\left(\left\langle\omega, e_{n}\right\rangle\right)
$$

Lemma 1.3 (fractional Wiener-Ito chaos expansion theorem) Let $X \in L^{2}(\mu)$. Then there exists constants $c_{\alpha} \in \mathbf{R}$ and $\alpha \in I$ such that:

$$
X(\omega)=\sum_{\alpha \in I} c_{\alpha} H_{\alpha}(\omega) \quad\left(\text { in } L^{2}(\mu)\right)
$$

and

$$
\|X\|_{L^{2}(\mu)}^{2}=\sum_{\alpha \in I} \alpha ! c_{\alpha}^{2}
$$

where $\alpha !:=\alpha_{1} ! \alpha_{2} ! \ldots \alpha_{n} !$ 
Define $(S)_{H}^{*}$ the set of all formal expansions

such that

$$
G(\omega)=\sum_{\alpha \in I} c_{\alpha} H_{\alpha}(\omega)
$$

$$
\|G\|_{H,-q}^{2}=\sum_{\alpha \in I} \alpha ! c_{\alpha}^{2}(2 \mathbf{N})^{-\alpha q}<\infty \text { for some } q \in \mathbf{N}
$$

If $F(\omega)=\sum_{\alpha \in I} a_{\alpha} \mathrm{H}_{\alpha}(\omega) \in(S)_{H}^{*}$ and $G(\omega)=\sum_{\alpha \in I} b_{\alpha} \mathrm{H}_{\alpha}(\omega) \in(S)_{H}^{*}$ we define the Wick product of $F$ and $G$ by

$$
(F \diamond G)(\omega)=\sum_{\alpha, \beta \in I} a_{\alpha} b_{\beta} \mathrm{H}_{\alpha+\beta}(\omega)
$$

Lemma 1.4 Let $f, g \in L_{\phi}^{2}(\mathbf{R})$. We have that

$$
\varepsilon(f) \diamond \varepsilon(g)=\varepsilon(f+g)=\varepsilon(f) \varepsilon(g) e^{-\langle f, g\rangle_{\phi}}
$$

The fractional white noise $W_{H}(t)$ at time $t$ is defined by:

$$
W_{H}(t)=\sum_{i=1}^{\infty}\left[\int_{R} e_{i}(v) \phi(t, v) d v\right] \int_{R} e_{i}(t) d B_{H}(t)
$$

We have that $W_{H}(t) \in(S)_{H}^{*}$ and $\frac{d}{d t} B_{H}(t)=W_{H}(t)$ in $(S)_{H}^{*}$.

If $Y: \mathbf{R} \rightarrow(S)_{H}^{*}$ is a function such that $Y(t) \diamond W_{H}(t)$ is integrable in $(S)_{H}^{*}$ the fractional Ito integral is defined by:

$$
\int_{R} Y(t) d B_{H}(t):=\int_{R} Y(t) \diamond W_{H}(t) d t
$$

Lemma 1.5 (Geometric fractional Brownian motion) Consider the fractional differential equation:

$$
d X(t)=\mu X(t) d t+\sigma X(t) d B_{H}(t), \quad X(0)=x
$$

We have that:

$$
X(t)=x \exp \left(\sigma B_{H}(t)+\mu t-\frac{1}{2} \sigma^{2} t^{2 H}\right)
$$

For the definition of the space $\mathrm{L}_{\phi}^{1,2}$ and of the Malliavin derivatives $D_{s} X, D_{s}^{\phi} X$ consult $\mathrm{Hu}$ and Oksendal (2000). equation:

Lemma 1.6 (Fractional Ito formula) Consider the fractional differential

$$
d X(t)=\mu(t, \omega) d t+\sigma(t, \omega) d B_{H}(t), \quad \mu, \sigma \in \mathrm{L}_{\phi}^{1,2}
$$

If $f \in C^{2}\left(\mathbf{R}_{+} \times \mathbf{R}\right)$ then we have: 


$$
\begin{aligned}
f(t, X(t))= & f(0, X(0))+\int_{0}^{t} \frac{\partial f}{\partial s}(s, X(s)) d s+\int_{0}^{t} \frac{\partial f}{\partial x}(s, X(s)) \mu(s) d s+ \\
& +\int_{0}^{t} \frac{\partial f}{\partial x}(s, X(s)) \sigma(s) d B_{H}(s)+\int_{0}^{t} \frac{\partial^{2} f}{\partial x^{2}}(s, X(s)) \sigma(s) D_{s}^{\phi} X(s) d s
\end{aligned}
$$

Lemma 1.7 (Fractional Girsanov formula) Let $T>0$ and $\gamma$ a continuos function with supp $\gamma \subset[0, T]$ and $K$ a function with supp $K \subset[0, T]$ such that

$$
\langle K, \gamma\rangle_{\phi}=\langle\gamma, f\rangle_{L^{2}}, \forall f \operatorname{supp} f \subset[0, T]
$$

Define a probability measure $\tilde{\mu}$ on the $\sigma$ - algebra $\mathrm{F}_{T}{ }^{H}=\mathrm{B}\left(B_{H}(s), s \leq T\right)$ by:

$$
\frac{d \tilde{\mu}}{d \mu}=\exp ^{\diamond}(-\langle\omega, K\rangle)
$$

Then $\tilde{B}_{H}(t)=B_{H}(t)+\int_{0}^{t} \gamma_{s} d s$ is a fractional Brownian motion under $\tilde{\mu}$.

Denote $\hat{L}_{\phi}^{2}\left(\mathbf{R}^{n}\right)$ the space of function that are symmetric with respect to its $n$ variables and $\|f\|_{L^{2}\left(R^{n}\right)}^{2}<\infty$.

We define the iterated integral:

$$
I_{n}(f):=\int_{R^{n}} f d B_{H}{ }^{\otimes n}:=n ! \int_{s_{1}<\ldots<s_{n}} f\left(s_{1}, \ldots, s_{n}\right) d B_{H}\left(s_{1}\right) \ldots d B_{H}\left(s_{n}\right)
$$

Lemma 1.8 (fractional Wiener-Ito chaos expansion theorem in terms of iterated integrals) Let $X \in L^{2}(\mu)$. Then there exists $f_{n} \in \hat{L}_{\phi}^{2}\left(\mathbf{R}^{n}\right)$ such that:

$$
X(\omega)=\sum_{n=0}^{\infty} I_{n}\left(f_{n}\right) \quad\left(\text { in } L^{2}(\mu)\right)
$$

and

$$
\|X\|_{L^{2}(\mu)}^{2}=\sum_{n=0}^{\infty} n !\left\|f_{n}\right\|_{L^{2}\left(R^{n}\right)}^{2} .
$$

We say that a formal expansion

$$
G=\sum_{n=0}^{\infty} \int_{R^{n}} g_{n} d B_{H}{ }^{\otimes n}, \quad g_{n} \in \hat{L}_{\phi}^{2}\left(\mathbf{R}^{n}\right)
$$

belongs to the space $\mathbf{G}_{-q}, q \in \mathbf{N}$ if

$$
\|G\|_{-q}^{2}=\sum_{n=0}^{\infty} n !\left\|g_{n}\right\|_{L^{2}\left(R^{n}\right)}^{2} e^{-2 q n}<\infty
$$

Let $\mathrm{G}^{*}=\bigcup_{q \in N} \mathrm{G}_{-q}$. We have that $L^{2}(\mu) \subset \mathrm{G}^{*}$ 
Let $G=\sum_{n=0}^{\infty} \int_{R^{n}} g_{n} d B_{H}{ }^{\otimes n} \in \mathrm{G}^{*}$. We define the quasi-conditional expectation of $G$ with respect to $\mathrm{F}_{t}{ }^{H}=\mathrm{B}\left(B_{H}(s), s \leq t\right)$ by:

$$
\widetilde{E}_{t}[G]:=\widetilde{E}\left[G \mid \mathrm{F}_{t}{ }^{H}\right]:=\sum_{n=0}^{\infty} \int_{R^{n}} g_{n}(s) \chi_{0 \leq s \leq t}(s) d B_{H}{ }^{\otimes n}(s)
$$

\section{Lemma 1.9}

a) Let $F \in \mathrm{G}^{*}$. We have that $\tilde{E}_{t}[F] \in \mathrm{G}^{*}$

b) Let $F, G \in \mathrm{G}^{*}$. We have that $\widetilde{E}_{t}[F \diamond G]=\widetilde{E}_{t}[F] \diamond \widetilde{E}_{t}[G]$

c) Let $F \in L^{2}(\mu)$. $\tilde{E}_{t}[F]=F \Leftrightarrow F$ is $\mathrm{F}_{t}{ }^{H}$ - measurable

We say that a $\mathrm{F}_{t}{ }^{H}$ - adapted stochastic process $M(t, \omega)$ is a quasi-martingale if $M(t) \in \mathrm{G}^{*}, \forall t$ and $\widetilde{E}_{s}[M(t)]=M(s), \forall t \geq s$.

\section{Lemma 1.10}

a) $B_{H}(t)$ is a quasi-martingale

b) Let $f \in L_{\phi}^{2}(\mathbf{R})$ and $\varepsilon(t):=\exp \left(\int_{0}^{t} f(s) d B_{H}(s)-\frac{1}{2}\left|f \chi_{[0, t]}\right|_{\phi}^{2}\right)$. We have that $\varepsilon(t)$ is a quasi-martingale

c) Let $f \in \mathrm{L}_{\phi}^{1,2}$ and $M(t):=\int_{0}^{t} f(s, \omega) d B_{H}(s)$. We have that $M(t)$ is a quasimartingale

Lemma 1.11 (fractional Clark-Ocone theorem)

a) Let $F \in \mathrm{G}^{*}$ and $F$ is $\mathrm{F}_{T}{ }^{H}$ - measurable. Then $\tilde{E}_{t}\left[D_{t} F\right] \in \mathrm{G}^{*}$ and

$$
F(\omega)=E[F]+\int_{0}^{T} \tilde{E}_{t}\left[D_{t} F\right] \diamond W_{H}(t)
$$

b) Let $F \in L^{2}(\mu)$ and $F$ is $\mathrm{F}_{T}{ }^{H}$ - measurable. Then $\tilde{E}_{t}\left[D_{t} F\right] \in \mathrm{L}_{\phi}^{1,2}$ and

$$
F(\omega)=E[F]+\int_{0}^{T} \tilde{E}_{t}\left[D_{t} F\right] d B_{H}(t)
$$




\section{Some results regarding the quasi-conditional expectation}

Let $(\Omega, \mathrm{K}, \mu)$ a probability field such that $B_{H}(t, \omega)$ is a fractional Brownian motion with respect to $\mu$.

\section{Theorem 3.1}

For every $0<t<T$ and $\lambda \in \mathbf{C}$ we have $\widetilde{E}_{t}\left[e^{\lambda B_{H}(T)}\right]=e^{\lambda B_{H}(t)+\frac{\lambda^{2}}{2}\left(T^{2 H}-t^{2 H}\right)}$

Proof:

Consider the fractional differential equation:

$$
d X(t)=\lambda X(t) d B_{H}(t), \quad X(0)=1
$$

Using Lemma 1.5 we have that:

$$
X(t)=\exp \left(\lambda B_{H}(t)-\frac{1}{2} \lambda^{2} t^{2 H}\right)
$$

Since

$$
X(t)=\int_{0}^{t} \lambda X(s) d B_{H}(s)
$$

using Lemma $1.10 \mathrm{c}$ it follows that

$$
\tilde{E}_{t}[X(T)]=X(t)
$$

or

$$
\tilde{E}_{t}\left[e^{\lambda B_{H}(T)}\right]=e^{\lambda B_{H}(t)+\frac{\lambda^{2}}{2}\left(T^{2 H}-t^{2 H}\right)}
$$

q.e.d.

\section{Theorem 3.2}

Let $f$ be a function such that $E\left[f\left(B_{H}(T)\right)\right]<\infty$. Then for every $t \leq T$

$$
\tilde{E}_{t}\left[f\left(B_{H}(T)\right)\right]=\int_{R} \frac{1}{\sqrt{2 \pi\left(T^{2 H}-t^{2 H}\right)}} \exp \left(-\frac{\left(x-B_{H}(t)\right)^{2}}{2\left(T^{2 H}-t^{2 H}\right)}\right) f(x) d x
$$

Proof:

Let $\hat{f}$ be the Fourier transform of $f$ :

$$
\hat{f}(\xi)=\int_{R} e^{-i x \xi} f(x) d x
$$


Then $f$ is the inverse Fourier transform of $\hat{f}$ :

$$
f(x)=\frac{1}{2 \pi} \int_{R} e^{i x \xi} \hat{f}(\xi) d \xi
$$

We have that:

$$
f\left(B_{H}(T)\right)=\frac{1}{2 \pi} \int_{R} e^{i B_{H}(T) \xi} \hat{f}(\xi) d \xi
$$

If follows that:

$$
\begin{aligned}
\tilde{E}_{t}\left[f\left(B_{H}(T)\right)\right] & =\tilde{E}_{t}\left[\frac{1}{2 \pi} \int_{R} e^{i \xi B_{H}(T)} \hat{f}(\xi) d \xi\right] \\
& =\frac{1}{2 \pi} \int_{R} \tilde{E}_{t}\left[e^{i \xi B_{H}(T)}\right] \hat{f}(\xi) d \xi \\
& =\frac{1}{2 \pi} \int_{R} e^{i \xi B_{H}(t)-\frac{\xi^{2}}{2}\left(T^{2 H}-t^{2 H}\right)} \hat{f}(\xi) d \xi \\
& =h\left(B_{H}(t)\right)
\end{aligned}
$$

where $h$ is the inverse Fourier transform of the product between $e^{-\frac{\xi^{2}}{2}\left(T^{2 H}-t^{2 H}\right)}$ and $\hat{f}(\xi)$.

But the first function is the Fourier transform of

$$
n_{t, T}(x)=\frac{1}{\sqrt{2 \pi\left(T^{2 H}-t^{2 H}\right)}} \exp \left(-\frac{x^{2}}{2\left(T^{2 H}-t^{2 H}\right)}\right)
$$

Using the fact that the Fourier transform of a convolution is the product of the Fourier transform of the two functions it follows that

$$
h\left(B_{H}(t)\right)=\int_{R} n_{t, T}\left(B_{H}(t)-y\right) f(y) d y
$$

q.e.d

\section{Corollary 3.3}

Let $A \in \mathrm{B}(\mathbf{R})$. Then

$$
\tilde{E}_{t}\left[\chi_{A}\left(B_{H}(T)\right)\right]=\int_{A} \frac{1}{\sqrt{2 \pi\left(T^{2 H}-t^{2 H}\right)}} \exp \left(-\frac{\left(x-B_{H}(t)\right)^{2}}{2\left(T^{2 H}-t^{2 H}\right)}\right) d x \quad 3.6
$$


Let $\theta \in \mathbf{R}$. Consider the process

$$
B_{H}^{*}(t)=B_{H}(t)+\theta t^{2 H}=B_{H}(t)+\int_{0}^{t} 2 H \theta \tau^{2 H-1} d \tau, \quad 0 \leq t \leq T
$$

Lemma 1.7 assures us that there is a measure $\mu^{*}$ such that $B_{H}^{*}(t)$ is a fractional Brownian motion under $\mu^{*}$.

We will denote $\tilde{E}_{t}^{*}[\cdot]$ the quasi-conditional expectation with respect to $\mu^{*}$.

Consider

$$
Z(t)=\varepsilon\left(-\theta \chi_{[0, t]}\right)=\exp \left(-\theta B_{H}(t)-\frac{\theta^{2}}{2} t^{2 H}\right)
$$

\section{Theorem 3.4}

Let $f$ be a function such that $E\left[f\left(B_{H}(T)\right)\right]<\infty$. Then for every $t \leq T$

$$
\widetilde{E}_{t}^{*}\left[f\left(B_{H}(T)\right)\right]=\frac{1}{Z(t)} \widetilde{E}_{t}\left[f\left(B_{H}(T)\right) Z(T)\right]
$$

\section{Proof:}

Again we will denote by $\hat{f}$ the Fourier transform of $f$.

We have

$$
\begin{aligned}
& \tilde{E}_{t}\left[f\left(B_{H}(T)\right) Z(T)\right]=\tilde{E}_{t}\left[\frac{1}{2 \pi} \int_{R} e^{(i \xi-\theta) B_{H}(T)-\frac{\theta^{2}}{2} T^{2 H}} \hat{f}(\xi) d \xi\right] \\
& =\frac{1}{2 \pi} e^{\frac{\theta^{2}}{2} T^{2 H}} \int_{R} \widetilde{E}_{t}\left[e^{(i \xi-\theta) B_{H}(T)}\right] \hat{f}(\xi) d \xi \\
& =\frac{1}{2 \pi} e^{-\frac{\theta^{2}}{2} T^{2 H}} \int_{R} e^{(i \xi-\theta) B_{H}(t)+\left(-\frac{\xi^{2}}{2}-i \xi \theta+\frac{\theta^{2}}{2}\right)\left(T^{2 H}-t^{2 H}\right)} \hat{f}(\xi) d \xi \\
& =Z(t) \frac{1}{2 \pi} \int_{R} e^{i \xi B_{H}(t)+\left(-\frac{\xi^{2}}{2}-i \xi \theta\right)\left(T^{2 H}-t^{2 H}\right)} \hat{f}(\xi) d \xi
\end{aligned}
$$


On the other hand

$$
\begin{aligned}
\widetilde{E}_{t}^{*}\left[f\left(B_{H}(T)\right)\right] & =\widetilde{E}_{t}^{*}\left[\frac{1}{2 \pi} \int_{R} e^{i \xi\left(B_{H}^{*}(T)-\theta T^{2 H}\right)} \hat{f}(\xi) d \xi\right] \\
& =\frac{1}{2 \pi} \int_{R} \widetilde{E}_{t}^{*}\left[e^{i \xi B_{H}^{*}(T)}\right] e^{-i \xi \theta T^{2 H}} \hat{f}(\xi) d \xi \\
& =\frac{1}{2 \pi} \int_{R} e^{i \xi B_{H}^{*}(t) \frac{\xi^{2}}{2}\left(T^{2 H}-t^{2 H}\right)} e^{-i \xi \theta T^{2 H}} \hat{f}(\xi) d \xi \\
& =\frac{1}{2 \pi} \int_{R} e^{i \xi\left(B_{H}(t)+\theta t^{2 H}\right)-\frac{\xi^{2}}{2}\left(T^{2 H}-t^{2 H}\right)} e^{-i \xi \theta T^{2 H}} \hat{f}(\xi) d \xi \\
& =\frac{1}{2 \pi} \int_{R} e^{i \xi B_{H}(t)+\left(-\frac{\xi^{2}}{2}-i \xi \theta\right)\left(T^{2 H}-t^{2 H}\right)} \hat{f}(\xi) d \xi
\end{aligned}
$$

The result follows from 3.10 and 3.11

q.e.d. 


\section{Applications to Mathematical Finance}

Consider a fractional Black-Sholes market that has two investment possibilities:

1. a money market account:

$$
d M(t)=r M(t) d t, \quad M(0)=1, \quad 0 \leq t \leq T
$$

where $r$ represent the constant riskless interest rate.

2. a stock whose price satisfies the equation:

$$
d S(t)=\delta S(t) d t+\sigma S(t) d \bar{B}_{H}(t), \quad S(0)=S>0,0 \leq t \leq T
$$

where $\delta, \sigma \neq 0$ are constants.

$\mathrm{Hu}$ and Oksendal (2000) have shown that this market does not have arbitrage and is complete.

Under the risk-neutral measure $(\mu)$ we have that:

$$
d S(t)=r S(t) d t+\sigma S(t) d B_{H}(t), \quad S(0)=S>0,0 \leq t \leq T
$$

We will denote by $\tilde{E}_{t}[\cdot]$ the quasi-conditional expectation with respect to the risk-neutral measure.

Theorem 4.1 (fractional risk-neutral evaluation) is given by

The price at every $t \in[0, T]$ of a bounded $\mathrm{F}_{T}{ }^{H}$ - measurable claim $F \in L^{2}(\mu)$

$$
F(t)=e^{-r(T-t)} \tilde{E}_{t}[F]
$$

\section{Proof:}

Since the market is complete there is a replicating portfolio of the claim $(m(t), s(t))$ whose value is:

$$
F(t)=m(t) M(t)+s(t) S(t)
$$

and

$$
F(T)=F
$$

We have that

$$
\begin{aligned}
d F(t) & =m(t) d M(t)+s(t) d S(t) \\
& =r F(t) d t+\sigma s(t) S(t) d B_{H}(t)
\end{aligned}
$$


By multiplying with $e^{-r t}$ and integrating it follows that

$$
e^{-r t} F(t)=F(0)+\int_{0}^{t} e^{-r \tau} \sigma s(\tau) S(\tau) d B_{H}(\tau), 0 \leq t \leq T
$$

By the fractional Clark-Ocone theorem (Lemma 1.11) we have that

$$
e^{-r T} F=E[F]+e^{-r T} \int_{0}^{T} \tilde{E}_{\tau}\left[D_{\tau} F\right] d B_{H}(\tau)
$$

From the completeness of the market we get

$$
\tilde{E}_{\tau}\left[D_{\tau} F\right]=e^{r(T-\tau)} \sigma s(\tau) S(\tau), 0 \leq \tau \leq T
$$

So we have that

$$
e^{-r T} F=E[F]+\int_{0}^{T} e^{-r \tau} \sigma s(\tau) S(\tau) d B_{H}(\tau)
$$

It follows that

$$
\tilde{E}_{t}\left[e^{-r T} F\right]=E[F]+\widetilde{E}_{t}\left[\int_{0}^{T} e^{-r \tau} \sigma s(\tau) S(\tau) d B_{H}(\tau)\right]
$$

Using Lemma 1.10 we get that

$$
\tilde{E}_{t}\left[e^{-r T} F\right]=E[F]+\int_{0}^{t} e^{-r \tau} \sigma s(\tau) S(\tau) d B_{H}(\tau)
$$

From 4.5 and 4.9 we have that

$$
F(t)=e^{-r(T-t)} \tilde{E}_{t}[F]
$$

Theorem 4.2 (fractional Black-Sholes formula)

The price at every $t \in[0, T]$ of an european call option with strike price $K$ and maturity $T$ is given by

$$
C(t, S(t))=S(t) N\left(d_{1}\right)-K e^{-r \cdot(T-t)} N\left(d_{2}\right)
$$

where

$$
\begin{aligned}
& d_{1}=\frac{\ln \left(\frac{S(t)}{K}\right)+r(T-t)+\frac{\sigma^{2}}{2}\left(T^{2 H}-t^{2 H}\right)}{\sigma \sqrt{T^{2 H}-t^{2 H}}} \text { and } \\
& d_{2}=\frac{\ln \left(\frac{S(t)}{K}\right)+r(T-t)-\frac{\sigma^{2}}{2}\left(T^{2 H}-t^{2 H}\right)}{\sigma \sqrt{T^{2 H}-t^{2 H}}}
\end{aligned}
$$

and $N(\cdot)$ is the cumulative probability of the standard normal distribution. 
Proof:

We have that

$$
\begin{aligned}
C(t, S(t)) & =\tilde{E}_{t}\left[e^{-r \cdot(T-t)} \max ((S(T)-K), 0)\right] \\
& =\tilde{E}_{t}\left[e^{-r \cdot(T-t)} S(T) \chi_{\{S(T)>K\}}\right]-X e^{-r \cdot(T-t)} \tilde{E}_{t}\left[\chi_{\{S(T)>K\}}\right] \\
& =e^{-r \cdot(T-t)} \tilde{E}_{t}\left[S(T) \chi_{\{S(T)>K\}}\right]-X e^{-r \cdot(T-t)} \tilde{E}_{t}\left[\chi_{\{S(T)>K\}}\right]
\end{aligned}
$$

But if we denote by

$$
d_{2}^{*}=\frac{\ln (K / S)-r T+\frac{1}{2} \sigma^{2} T^{2 H}}{\sigma}
$$

we get

$$
\begin{aligned}
\tilde{E}_{t}\left[\chi_{\{S(T)>K\}}=\right. & \tilde{E}_{t}\left[\chi_{\left\{x>d_{2}^{*}\right\}}\left(B_{H}(T)\right)\right] \\
= & \int_{d_{2}^{*}}^{\infty} \frac{1}{\sqrt{2 \pi\left(T^{2 H}-t^{2 H}\right)}} \exp \left(-\frac{\left(x-B_{H}(t)\right)^{2}}{2\left(T^{2 H}-t^{2 H}\right)}\right) d x \\
= & \int^{\infty} \frac{1}{\sqrt{2 \pi}} \exp \left(-\frac{z^{2}}{2}\right) d z \\
& \frac{d^{*}{ }^{-} B_{H}(t)}{\sqrt{T^{2 H}-t^{2 H}}} \\
= & \int_{-\infty}^{\sqrt{T^{2 H}-d_{2}^{2 H}}} \frac{1}{\sqrt{2 \pi}} \exp \left(-\frac{z^{2}}{2}\right) d z \\
= & N\left(d_{2}\right)
\end{aligned}
$$

Consider the process

$$
B_{H}^{*}(t)=B_{H}(t)-\sigma t^{2 H}, 0 \leq t \leq T
$$

Lemma 1.7 assures us that there is a measure $\mu^{*}$ such that $B_{H}^{*}(t)$ is a fractional Brownian motion under $\mu^{*}$.

We will denote

$$
Z(t)=\exp \left(\sigma B_{H}(t)-\frac{\sigma^{2}}{2} t^{2 H}\right)
$$


Using Theorem 3.4 we have that

$$
\begin{aligned}
\tilde{E}_{t}\left[S(T) \chi_{\{S(T)>K\}}\right] & =e^{r T} \tilde{E}_{t}\left[Z(T) \chi_{\left\{x>d_{2}^{*}\right\}}\left(B_{H}(T)\right)\right] \\
& =e^{r T} Z(t) \tilde{E}_{t}^{*}\left[\chi_{\left\{x>d_{2}^{*}\right\}}\left(B_{H}(T)\right)\right] \\
& =e^{r T} Z(t) \tilde{E}_{t}^{*}\left[\chi_{\left\{x>d_{2}^{*}\right\}}\left(B_{H}(T)\right)\right] \\
& =e^{r T} Z(t) \tilde{E}_{t}^{*}\left[\chi_{\{S(T)>K\}}\right]
\end{aligned}
$$

But

$$
\begin{aligned}
\ln (S(T)) & =\ln S+r T-\frac{\sigma^{2}}{2} T^{2 H}+\sigma B_{H}(T) \\
& =\ln S+r T+\frac{\sigma^{2}}{2} T^{2 H}+\sigma B_{H}^{*}(T)
\end{aligned}
$$

If we denote

$$
d_{1}^{*}=\frac{\ln (K / S)-r T-\frac{1}{2} \sigma^{2} T^{2 H}}{\sigma}
$$

we get

$$
\begin{aligned}
\tilde{E}_{t}^{*}\left[\chi_{\{S(T)>K\}}\right]= & \tilde{E}_{t}^{*}\left[\chi_{\left\{x>d_{1}^{*}\right\}}\left(B_{H}^{*}(T)\right)\right] \\
= & \int_{d_{1}^{*}}^{\infty} \frac{1}{\sqrt{2 \pi\left(T^{2 H}-t^{2 H}\right)}} \exp \left(-\frac{\left(x-B_{H}^{*}(t)\right)^{2}}{2\left(T^{2 H}-t^{2 H}\right)}\right) d x \\
= & \int_{d_{1}^{*}-B_{H}^{*}(t)}^{\infty} \frac{1}{\sqrt{2 \pi}} \exp \left(-\frac{z^{2}}{2}\right) d z \\
& \frac{B_{H}^{2 H}-t^{2 H}}{\sqrt{T^{2 H}-d_{1}^{*}}} \\
= & \int_{-\infty}^{-t^{2 H}} \frac{1}{\sqrt{2 \pi}} \exp \left(-\frac{z^{2}}{2}\right) d z \\
= & N\left(d_{1}\right)
\end{aligned}
$$


So

$$
\begin{aligned}
\tilde{E}_{t}\left[S(T) \chi_{\{S(T)>K\}}\right] & =e^{r T} Z(t) N\left(d_{1}\right) \\
& =e^{r T} e^{-r t} S(t) N\left(d_{1}\right)
\end{aligned}
$$

From 4.11 and 4.16 we get the price of the option.

q.e.d

Theorem 4.3 (fractional Black-Sholes equation)

The price of a derivative on the stock price with a bounded payoff $f(S(T))$ is given by $D(t, S(t))$, where $D(t, S)$ is the solution of the PDE:

$$
\begin{aligned}
& \frac{\partial D}{\partial t}+H \sigma^{2} t^{2 H-1} S^{2} \frac{\partial^{2} D}{\partial S^{2}}+r S \frac{\partial D}{\partial S}-r D=0 \\
& D(T, S)=f(S)
\end{aligned}
$$

\section{Proof:}

From Theorem 4.1 and Theorem 3.2 it follows that the price of the derivative at a moment $t$ is a function of $t$ and $S(t)$.

As in the classical Black-Sholes model we consider a portfolio that contains a derivative and $-\Delta$ stock.

The value of this portfolio is

$$
\Pi(t)=D(t, S(t))-\Delta S(t)
$$

Under the market measure $\bar{\mu}$ using fractional Ito formula (Lemma 1.6) and the fact that

and

$$
\begin{aligned}
D_{\tau} S(\tau) & =S(\tau) D_{u}\left(\sigma B_{H}(\tau)+\delta \tau-\frac{\sigma^{2}}{2} \tau^{2 H}\right) \\
& =S(\tau) D_{u}\left(\sigma B_{H}(\tau)\right) \\
& =\sigma S(\tau) \chi_{[0, \tau]}(u)
\end{aligned}
$$

$$
D_{\tau}^{\phi} S(\tau)=\sigma S(\tau) \int_{0}^{\tau} \phi(\tau, u) d u=\sigma H S(\tau) \tau^{2 H-1}
$$

we get that

$$
\begin{aligned}
d \Pi(t) & =d D(t, S(t))-\Delta d S(t) \\
& =\left(\frac{\partial D}{\partial t}+H \sigma^{2} t^{2 H-1} S^{2} \frac{\partial^{2} D}{\partial S^{2}}+\delta S \frac{\partial D}{\partial S}-\Delta \delta S\right) d t+\left(\sigma S \frac{\partial D}{\partial S}-\Delta \sigma S\right) d \bar{B}_{H}(t)
\end{aligned}
$$


We want this portfolio to be riskless. So

$$
\Delta=\frac{\partial D}{\partial S} \quad \text { and } \quad d \Pi(t)=r \Pi(t) d t
$$

It follows that evaluation equation is given by:

$$
\frac{\partial D}{\partial t}+r S \frac{\partial D}{\partial S}+H \sigma^{2} t^{2 H-1} S^{2} \frac{\partial^{2} D}{\partial S^{2}}-r D=0
$$

\section{Remarks}

q.e.d

- $\quad$ As in the classical model the equation does not depend on $\delta$

- $\quad$ The price of the european call given by theorem Theorem 4.2 is the solution of the equation 4.17 with the boundary condition:

$$
D(t, S)=\max (S-K, 0)
$$

Theorem 4.5 (The Greeks)

The Greeks are given by:

$$
\begin{gathered}
\Delta=\frac{\partial C}{\partial S}=N\left(d_{1}\right) \\
\nabla=\frac{\partial C}{\partial K}=-e^{-r(T-t)} N\left(d_{2}\right) \\
\vartheta=\frac{\partial C}{\partial \sigma}=S f\left(d_{1}\right) \sqrt{T^{2 H}-t^{2 H}} \\
\rho=\frac{\partial C}{\partial r}=(T-t) K e^{-r(T-t)} N\left(d_{2}\right) \\
\Gamma=\frac{\partial^{2} C}{\partial S^{2}}=\frac{1}{S \sigma \sqrt{T^{2 H}-t^{2 H}}} f\left(d_{1}\right) \\
\Theta=\frac{\partial C}{\partial t}=-r K e^{-r(T-t)} N\left(d_{2}\right)-H t^{2 H-1} \frac{S \sigma}{\sqrt{T^{2 H}-t^{2 H}}} f\left(d_{1}\right)
\end{gathered}
$$

where

$$
f(z)=\frac{1}{\sqrt{2 \pi}} e^{-\frac{z^{2}}{2}}
$$

Proof:

We will first derive a general formula. Let $y$ be one of the influence factors.

We have

$$
\frac{\partial C}{\partial y}=\frac{\partial S}{\partial y} N\left(d_{1}\right)+S \frac{\partial N\left(d_{1}\right)}{\partial y}-\frac{\partial\left(K e^{-r \cdot(T-t)}\right)}{\partial y} N\left(d_{2}\right)-K e^{-r \cdot(T-t)} \frac{\partial N\left(d_{2}\right)}{\partial y}
$$


But

$$
\begin{aligned}
\frac{\partial N\left(d_{2}\right)}{\partial y} & =\frac{1}{\sqrt{2 \pi}} \exp \left(-\frac{d_{2}^{2}}{2}\right) \frac{\partial d_{2}}{\partial y}=\frac{1}{\sqrt{2 \pi}} \exp \left(-\frac{\left(d_{1}-\sigma \sqrt{T^{2 H}-t^{2 H}}\right)^{2}}{2}\right) \frac{\partial d_{2}}{\partial y} \\
& =\frac{1}{\sqrt{2 \pi}} \exp \left(-\frac{d_{1}^{2}}{2}\right) \exp \left(d_{1} \sigma \sqrt{T^{2 H}-t^{2 H}}\right) \exp \left(-\frac{\sigma^{2}\left(T^{2 H}-t^{2 H}\right)}{2}\right) \frac{\partial d_{2}}{\partial y} \\
& =\frac{1}{\sqrt{2 \pi}} \exp \left(-\frac{d_{1}^{2}}{2}\right) \exp \left(\ln \left(\frac{S(t)}{K}\right)+r(T-t)\right) \frac{\partial d_{2}}{\partial y} \\
& =\frac{1}{\sqrt{2 \pi}} \exp \left(-\frac{d_{1}^{2}}{2}\right) \frac{S(t)}{K} \exp (r(T-t)) \frac{\partial d_{2}}{\partial y}
\end{aligned}
$$

It follows that:

$$
\frac{\partial C}{\partial y}=\frac{\partial S}{\partial y} N\left(d_{1}\right)-\frac{\partial\left(K e^{-r \cdot(T-t)}\right)}{\partial y} N\left(d_{2}\right)+S f\left(d_{1}\right) \frac{\partial\left(\sigma \sqrt{T^{2 H}-t^{2 H}}\right)}{\partial y}
$$

Substituting in 4.21 we get the Greeks.

q.e.d

\section{Remarks}

The fractional Black-Sholes price of a european call option no longer depends only on $T-t$.

A reason may be the fact that the fractional Brownian motion has long memory. The price of an option at a moment $t \in[0, T]$ will depend on the stock price $S(t)$, but despite the classical Black-Sholes model, will take into consideration the evolution of the stock price in the period $[0, t]$. This influence is reflected in the fractional Black-Sholes formula by the Hurst parameter $H$.

Consider three moments $t_{1} \leq t_{2} \leq t \leq T$ and two options with maturity $T$ one of them written on $t_{1}$ and the other one on $t_{2}$. In the classical Black-Sholes model the prices of the two options at the moment $t$ were equal.

In the fractional Black-Sholes model the prices of the two options at the moment $t$ are no longer equal. Due to the long memory property, the price of the first option is also influenced by the evolution of the stock price in the period $\left[t_{1}, t_{2}\right]$. 


\section{References}

Decreusefond, L and A.S. Ustunel (1999). Stochastic analysis of the fractional Brownian motion . Potential Analysis ,10, 177-214

Duncan, T. E., Y. Hu and B. Pasik-Duncan (2000). Stochastic calculus for fractional Brownian motion. I. Theory. SIAM J. Control Optim. 38, 582-612.

Hu, Y. and B. Oksendal (2000). Fractional white noise calculus and application to Finance. Preprint, University of Oslo

Hu, Y., B. Oksendal, and A. Sulem (2000). Optimal consumption and portfolio in a Black-Scholes market driven by fractional Brownian motion. Preprint, University of Oslo 23/2000.

Lin, S.J. (1995). Stochastic analysis of fractional Brownian motion, fractional noises and applications. SIAM Review, 10, 422-437.

Rogers, L.C.G (1997). Arbitrage with fractional Brownian motion . Mathematical Finance, 7, 95-105 